\title{
Immune molecular profiling of a multiresistant primary prostate cancer with a neuroendocrine-like phenotype: a case report
}

\author{
Scott G. Williams 1,2,4, Han Xian Aw Yeang ${ }^{3,5}$, Catherine Mitchell ${ }^{4}$, Franco Caramia ${ }^{2}$, David J. Byrne ${ }^{4}$, \\ Stephen B. Fox ${ }^{4}$, Sue Haupt ${ }^{2,5}$, Ralf B. Schittenhelm ${ }^{6,7}$, Paul J. Neeson ${ }^{3,5}$, Ygal Haupt ${ }^{2,5,7 \dagger}$ \\ and Simon P. Keam ${ }^{2,3,5^{*}+}$
}

\begin{abstract}
Background: Understanding the drivers of recurrence in aggressive prostate cancer requires detailed molecular and genomic understanding in order to aid therapeutic interventions.We provide here a case report of histological, transcriptional, proteomic, immunological, and genomic features in a longitudinal study of multiple biopsies from diagnosis, through treatment, and subsequent recurrence.

Case presentation: Here we present a case study of a male in $70 \mathrm{~s}$ with high-grade clinically-localised acinar adenocarcinoma treated with definitive hormone therapy and radiotherapy. The patient progressed rapidly with rising PSA and succumbed without metastasis 52 months after diagnosis. We identified the expression of canonical histological markers of neuroendocrine PC (NEPC) including synaptophysin, neuron-specific enolase and thyroid transcription factor 1, as well as intact AR expression, in the recurrent disease only. The resistant disease was also marked by an extremely low immune infiltrate, extensive genomic chromosomal aberrations, and overactivity in molecular hallmarks of NEPC disease including Aurora kinase and E2F, as well as novel alterations in the CMYB pathway. We also observed that responses to both primary treatments (high dose-rate brachytherapy and androgen deprivation therapies) were consistent with known optimal responses_-ruling out treatment inefficacy as a factor in relapse.
\end{abstract}

Conclusions: These data provide novel insights into a case of locally recurrent aggressive prostate cancer harbouring NEPC pathology, in the absence of detected metastasis.

Keywords: Case report, Neuroendocrine, Localized, Brachytherapy, Hormone therapy

\section{Background}

Prostate cancer (PC) that recurs after primary treatment is a significant health issue. While the initial response rate to conventional therapy is high, most recurrences

\footnotetext{
*Correspondence: Simon.Keam@petermac.org

${ }^{\dagger}$ Ygal Haupt and Simon P. Keam: shared senior author.

${ }^{2}$ Tumor Suppression Laboratory, Peter MacCallum Cancer Centre,

Melbourne, Australia

Full list of author information is available at the end of the article
}

will progress to become resistant to ongoing salvage therapies, with high rates of lethal progression. More unusually, there are a subset of cancers that are refractory to therapies such as androgen deprivation therapy (ADT) de novo and carry a worse prognosis via rapid progression [1]. Identifying the molecular progression and the genomic features of these tumours will aid in early identification, treatment selection and novel therapeutic development. 
Here we present comprehensive histological, molecular and bioinformatic analysis of a case study of a male in their $70 \mathrm{~s}$ initially diagnosed with high grade but clinically localised prostate adenocarcinoma with local disease progression despite combined ADT and radiotherapy (using external beam radiation and brachytherapy), and died from progressive local disease-related complications in the absence of detectable metastases within 5 years of diagnosis.

In this study, we interrogated tissue samples available at baseline as well as multiple stages of the local progression of disease using a combination of histological, immunological, transcriptomic, proteomic, and genomic techniques. This revealed the emergence of an immune-excluded neuroendocrine-like tumour histology harbouring molecular and genomic characteristics of canonical neuroendocrine PC (NEPC). Despite these similarities, we have identified numerous novel molecular features which could improve our understanding of NEPC pathologies.

\section{Case presentation}

\section{Patient description and family history}

The case study patient was a male in their $70 \mathrm{~s}$ with preexisting conditions that were treated with metformin, candesartan and low-dose aspirin. A male relative died of cardiac disease in their $50 \mathrm{~s}$, and a female relative died from colon cancer in their $80 \mathrm{~s}$. The patient was a nonsmoker with only infrequent alcohol intake. An overview of diagnostic PSA metrics, treatments, pathology and sample acquisition timepoints are illustrated in Fig. 1.

\section{Diagnosis}

Patient had been undertaking PSA screening. In 2007, serum total PSA was $2.70 \mathrm{ng} / \mathrm{mL}$ (reference range $<4.51 \mathrm{ng} / \mathrm{mL}$ ) and subsequently was $2.98 \mathrm{ng} / \mathrm{mL}$ in 2011. He had developed mild lower urinary tract obstructive symptoms at that stage and proceeded to a transurethral resection of prostate with good symptomatic relief. Histology from that procedure was benign. In 2012, his PSA had risen to $5.29 \mathrm{ng} / \mathrm{mL}$ and then $12.81 \mathrm{ng} / \mathrm{mL}$ the following year. His urinary function was unchanged but he had a prostate suspicious for malignancy on digital rectal examination; clinical stage T2c. Histology from a transrectal ultrasound-guided biopsy of prostate showed every region contained prostatic acinar adenocarcinoma: Gleason Grade Group (GGG) 1 (right midzone, 2 mm of core involved), GGG 2 at right base, left apex and right transition zone (1, 2 and $7 \mathrm{~mm}$ respectively), GGG 4 at left midzone and right apex ( 4 and $1 \mathrm{~mm}$ respectively), and GGG 5 at left transition zone (10 $\mathrm{mm}$ involvement with perineural infiltration noted). A sample used for subsequent molecular analysis was collected at this stage
(Sample 1: Diagnostic; D). Staging with CT abdomen and pelvis and ${ }^{99 \mathrm{~m}} \mathrm{Tc}$-bone scan and reported as showing no evidence of metastases. Final clinical stage T2cN0M0.

\section{Commencement of treatment}

Recommended treatment was androgen deprivation therapy (ADT) for a total of 2 years with radical radiotherapy in the form of external beam RT + high-dose-rate brachytherapy (HDRBT). Goserelin $10.8 \mathrm{mg}$ implants provided androgen suppression throughout, and radiation therapy was commenced after 4 months of ADT using a sequence of HDRBT, a 2 week break followed by external beam RT (EBRT), another 2 week break and a further HDRBT fraction. HDRBT delivering $10 \mathrm{~Gy}$ in a single fraction to the prostate with a $3 \mathrm{~mm}$ margin in all directions apart from $0 \mathrm{~mm}$ posteriorly using CT planning. EBRT was to the prostate and seminal vesicles with a $10 \mathrm{~mm}$ margin in all direction except $6 \mathrm{~mm}$ posteriorly, delivering 46 Gy in 23 daily fractions over 4 weeks using CT planning, intensity modulated beams and daily image guidance with 3 intra-prostatic fiducial markers. Two tissue biopsy samples, each consisting of 4-5 16 g cores, were collected prior to each HDRBT delivery; HDRBT fraction 1 biopsy representing the effect of 4 months of ADT (Sample 2: Pre-HDRBT; $R T$-), and fraction 2 HDRBT biopsy representing the effect of ADT plus 10 Gy HDRBT+ 46 Gy EBRT (Sample 3: Post-HDRBT; RT+).

\section{Initial response and subsequent rapid development of castration-resistance}

Following 3 months of ADT, testosterone was fully suppressed $(<0.4 \mathrm{nmol} / \mathrm{L})$ and PSA reduced to $0.131 \mathrm{ng} /$ $\mathrm{mL}$. PSA was $0.066 \mathrm{ng} / \mathrm{mL} 1$ month after RT completion. Patient elected not to continue ADT subsequent to RT. Within 15 months of completion of RT, the PSA had risen to 2.41 and goserelin recommenced. Following a PSA nadir of $0.37 \mathrm{ng} / \mathrm{mL}$ at 3 months, the PSA rose rapidly. Addition of bicalutamide (50 $\mathrm{mg}$ daily) resulted in a PSA fall from 1.3 to $1.06 \mathrm{ng} / \mathrm{ml}$ at 3 months, followed by a rise to $4.0 \mathrm{ng} / \mathrm{mL}$ over the next 7 months. Clinical examination suggested ongoing disease in the prostate, and CT chest, abdomen and pelvis showed no evidence of metastases at that point. He was subsequently commenced on enzalutamide (160 mg daily) with no impact on PSA level.

\section{Disease progression and death}

Forty-four months since completion of RT, the patient presented with heavy haematuria, urinary obstruction and PSA of $19 \mathrm{ng} / \mathrm{mL}$. This required surgical intervention where a friable bleeding mass obstructing his urethra was resected transurethrally with initial good symptomatic effect. A component of this 


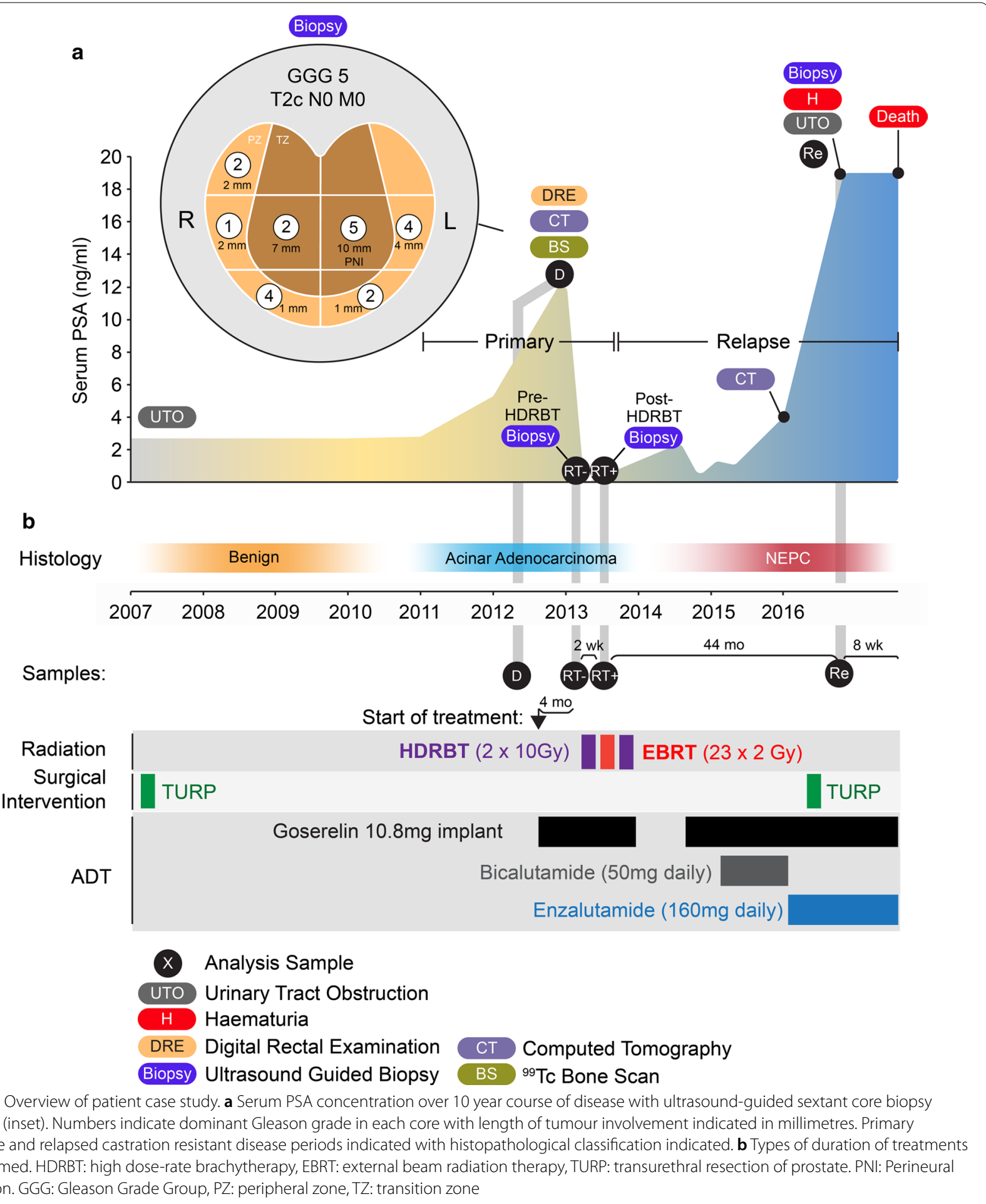

resection represented the final biopsy sample (Sample 4: Recurrence; $R e$ ). He represented with further bleeding a month later. Nephrostomy tubes were unable to protect his renal function and he deteriorated clinically and died as a consequence of locally progressive disease 52 months after RT without evidence of metastases by CT scan. Autopsy was not performed as the certifiable cause of death was not in question. Details of subsequent analysis methodologies can be found in Additional file 1. 


\section{Histopathological analysis: Onset of neuroendocrine-like histology at recurrence}

Histopathological examination evaluated differences in the series of patient samples and also defined tumour stroma and tumour regions for subsequent analyses (Fig. 2a). Acinar adenocarcinoma was identified in the initial diagnostic and subsequent pre- and post-HDRBT tissues, while poorly differentiated adenocarcinoma with areas of neuroendocrine differentiation was identified in the recurrence biopsy (Fig. 2b). Immunohistochemistry (IHC) for synaptophysin (SYN), neuron-specific enolase (NSE), thyroid transcription factor-1 (TTF-1), chromogranin (CG), CD56 and androgen receptor (AR) in the recurrence biopsy (Fig. 3) showed weak and/or patchy positive staining for SYN, NSE, TTF-1 and AR. CD56 exhibited non-specific staining and CG was negative. These results support the initial finding of neuroendocrine-like pathology with intact AR expression. Examination of all samples D, RT- and RT + using synaptophysin IHC (the most consistent marker in sample 4) showed no evidence of NEPC features, suggesting a late emergence of the NEPC-like phenotype (data not shown).

\section{Immune infiltrate is acutely affected by androgen deprivation, radiation and recurrence onset}

Studies of the immune context of neuroendocrine cancer are limited to non-existent. We hypothesised that the development of prostatic neuroendocrine-like pathologies could adversely affect the immune context of the tumour and could potentially provide insight into the characteristics of the disease. We therefore performed two-colour dual staining for CD3 (a marker of T cells) and CD45 (a general marker of leucocytes) (Fig. 4a). We used pathology markup of tumour and tumour stroma zones, as well as unbiased computational calculations of individual cells to generate an overall cell density for both cell types in either the tumour or tumour stroma relative to surface area. This was important as surface area studied varied between the samples (Table 1). Overall, $\mathrm{CD}^{+} \mathrm{T}$ cells were far more numerous than other $\mathrm{CD} 45^{+} \mathrm{CD}^{-}$leukocyte populations (Fig. 4b). With regards to the effects of acute androgen deprivation (Goserelin for 4 months: D to RT-), we observed that $\mathrm{CD}^{+} \mathrm{T}$ cell density was stimulated by up to fourfold (from 116.8 to 414.29 cells $/ \mathrm{mm}^{2}$ ) in tumour stroma, but halved (from $~ 508.5$ to 263.1 cells $/ \mathrm{mm}^{2}$ ) in tumour zones. The overall density of CD45+ CD3+ cells was largely unchanged (625.3 to 677.4 cells $/ \mathrm{mm}^{2}$ ) from a total tissue perspective. The minor non-T-cell CD45+ CD3had fewer changes, but did appear to have an increase in density in androgen-treated tissue (Fig. 4c). Radiation therapy (: RT- to RT+) had the most profound effect on immune cell density, with both populations being heavily reduced post-radiation 2 weeks following the last dose of external beam RT. Finally, we observed that the recurrence sample contained an even lower overall density of immune cells, with around tenfold less of all cell types when compared to the diagnostic biopsy. Overall, these data suggest that the recurrent PC microenvironment is profoundly immune excluded-regardless of tumour or its surrounding tumour stroma.

Due to limitations on the amount of tissue available for single stain IHC analyses, we used the whole transcriptomic and proteomic data to infer changes in the expression of genes and proteins for markers of major immune cell subsets (e.g. MS4A1/CD20 for B cells). The results, shown in Fig. 4d, suggested that CD163-expressing macrophages were highly expressed in both post-RT and recurrent tissues, the former being also observed in a recently published study of a larger group of patient receiving HDRBT [2]. B cells (assessed using MS4A1 or CD20) responded to early ADT but not to other treatments. Both dendritic cells (ITGAX/CD11c) and monocytes (CD14) were stimulated by radiation but plateaued in the recurrent biopsy. Natural killer cells (NCAM1/ CD56) did not change at any point in disease progression. Assessing T cells (either CD3 or CD4) using this method revealed notable increases after radiation, but drop to diagnostic levels in the recurrent biopsy. These transcriptional changes are not reflected in the previous single-colour stain performed earlier, most likely due to differences in the expression of transcripts and the encoded proteins.

\section{The transcriptional and proteomic landscape of case study specimens}

We performed transcriptomic and proteomic analysis of serial sections taken from all four FFPE biopsies $[3,4]$ to identify alterations in gene and protein levels (Fig. 5a). Early changes were consistent with the hormone therapy and radiation treatments. We compared the recurrence (sample 4) to the diagnostic specimen (sample 1) and

(See figure on next page.)

Fig. 2 Histopathological classification of tumour and tumour stroma zones identifies initial acinar adenocarcinoma and progression to small cell carcinoma. (A) Overview of H\&E stained sections used for histopathological and molecular analysis. Tissue zones comprising (i) tumour, (ii) tumour stroma, and (iii) mixed are indicated. Scale bar indicates $2 \mathrm{~mm}$. b High magnification H\&E stains of tumour stroma (one zone) and tumour (three zones) at each tissue collection timepoint. Scale bar indicates $100 \mu \mathrm{m}$ 

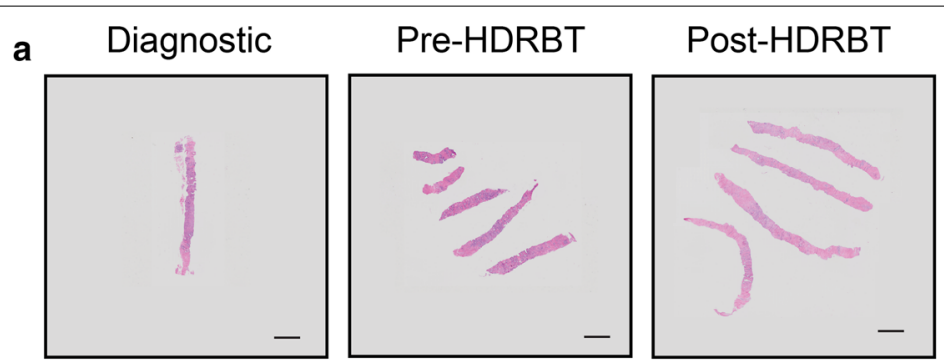

\section{Recurrence}
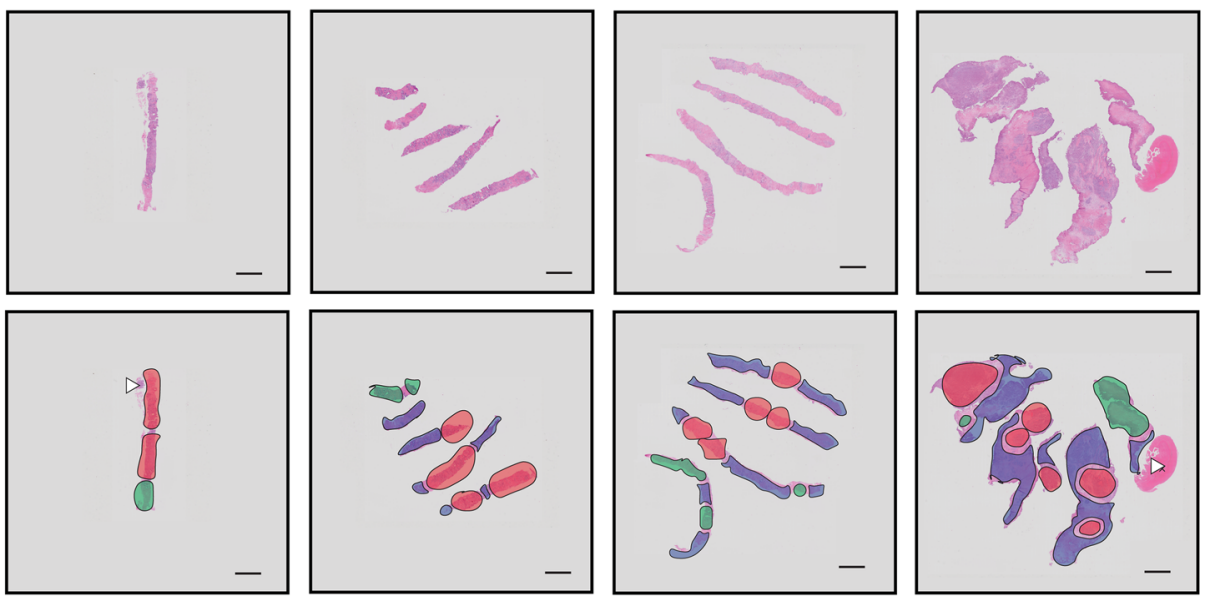

$\square$ Tumour stroma only

$\square$ Tumour only

$\square$ Mixed
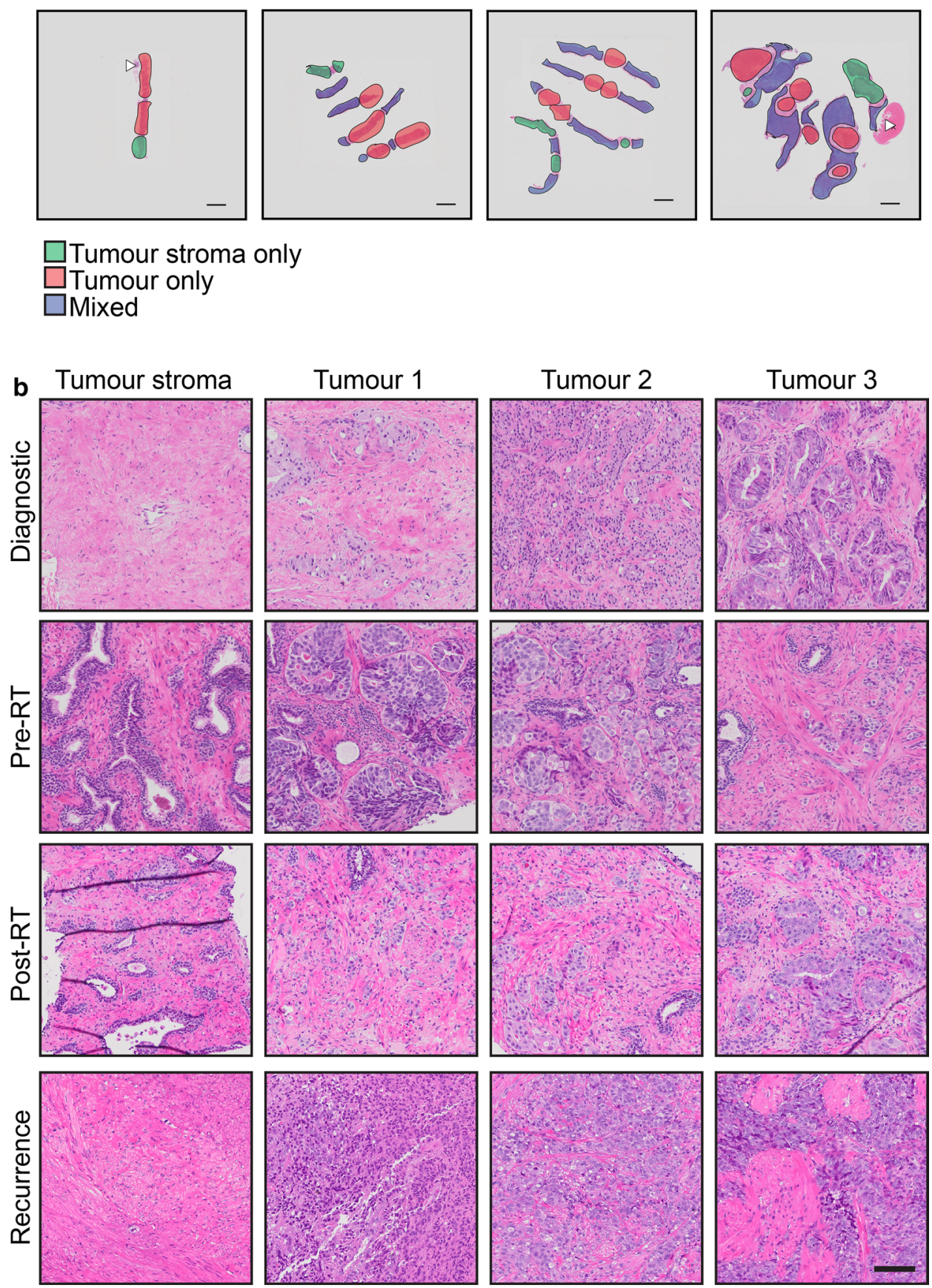


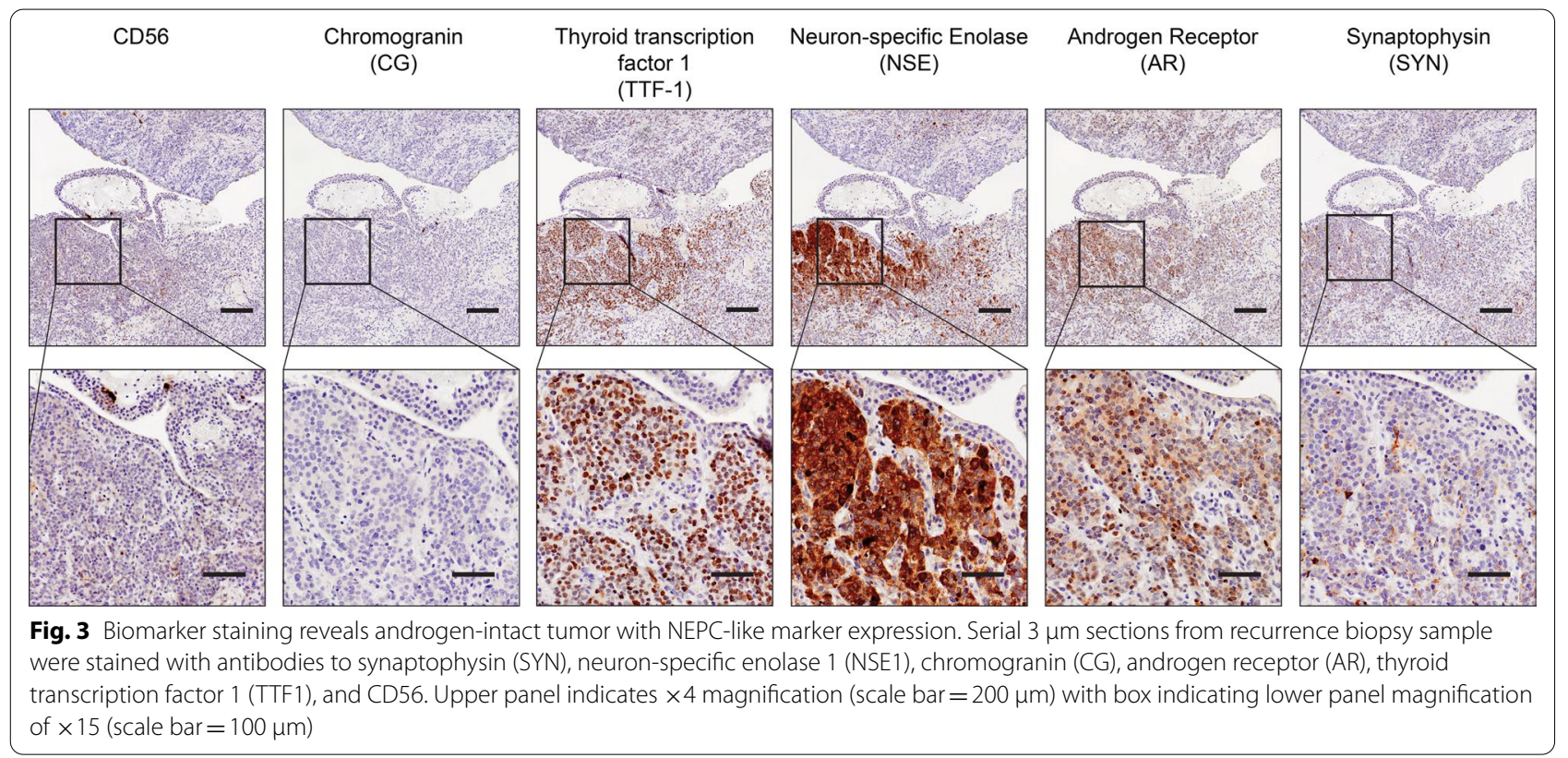

selected alterations common to both gene and protein analyses-identifying 11 upregulated and 8 downregulated gene/proteins (Fig. 5b). A description of these molecules and their known links to PC is outlined below.

\section{Upregulated molecules}

Within the eleven strongly upregulated molecules, we identified the DNA topoisomerase protein TOP $2 A$, which is a known early prognostic biomarker of several highrisk, aggressive and metastatic prostate cancer subtypes $[5,6]$. Interestingly, this protein is yet to be linked with neuroendocrine pathologies. We also identified the S100 Calcium Binding Proteins S100A8 and S100A9, which collectively form calprotectin-a faecal biomarker normally associated with intestinal inflammation and previously identified as a faecal marker of radiation-induced proctitis of the prostate [7]. The haemoglobin subunits $H B B$ and $H B A 2$ were also upregulated, likely consistent with the presence of a bleeding mass present at the time of biopsy and noted in the histopathology. Prostatic androgen receptor (AR) targets, including the Kinesinlike protein $K I F 1 A$, and GLUT3 (encoded by the $S L C 2 A 3$ gene) were also observed $[8,9]$, potentially consistent with the progressively androgen-independent biology of the disease. Karyopherin $\alpha 2$ (KPNA2) is a known predictor of biochemical recurrence in prostate cancer treated with radical prostatectomy [10]. Spondin-2, encoded by the SPON2 gene, has been proposed as a novel serum biomarker of the presence of $\mathrm{PC}$ and progression [11, 12]. The interferon stimulated genes $I S G 15$, and the $\mathrm{PH}$ domain-containing protein PHLDA2 were also identified.
These have no clear link with aggressive PC features in the literature; however the latter is known to be normally expressed in the adult prostate gland (Uniprot: data not shown).

\section{Downregulated molecules}

Amongst the downregulated proteins identified in recurrence tissue was the anterior gradient protein AGR2which has links with various PC pathologies. AGR2 has been shown to be commonly upregulated in primary PC adenocarcinoma, however lower expression levels are observed in metastasis and is highly predictive of biochemical recurrence (BCR) following RP [13]. Multidrug resistance protein 4 MRP4 $(A B C C 4)$ is an androgendriven gene that was also lost in the recurrence tissue and is known to be downregulated as PC progresses [14]. Arachidonate 15-lipoxygenase type II (ALOX15B), also known as 15-LOX2, is a prostate-specific enzyme also observed to be lost in the recurrent tissue. This enzyme has been shown to be lost in prostate cancer cells relative to normal prostate tissue $[15,16]$. Progastricsin, also known as Pepsinogen $C$, is encoded by the $P G C$ gene and also lost in the recurrence sample. Progastricsin is normally found in both normal and malignant prostatic tissue [17]. Its absence could be further evidence of the loss of conventional prostatic biology over the course of the disease. The plakophilin protein PKP1 was also lost and its expression, and it has been already demonstrated to be inversely correlated with Gleason grade in clinical PC adenocarcinomas [18]. 


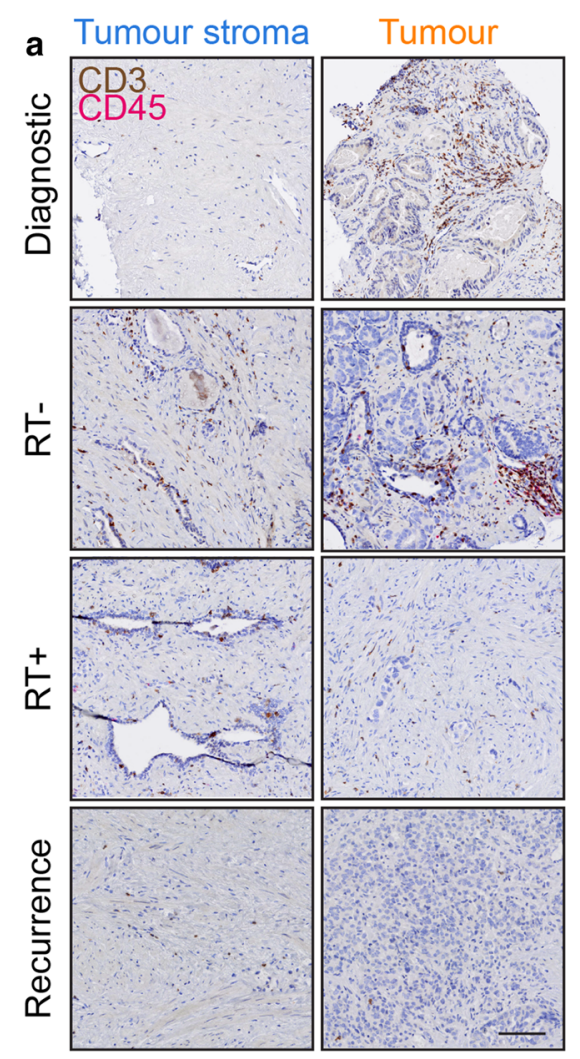

b

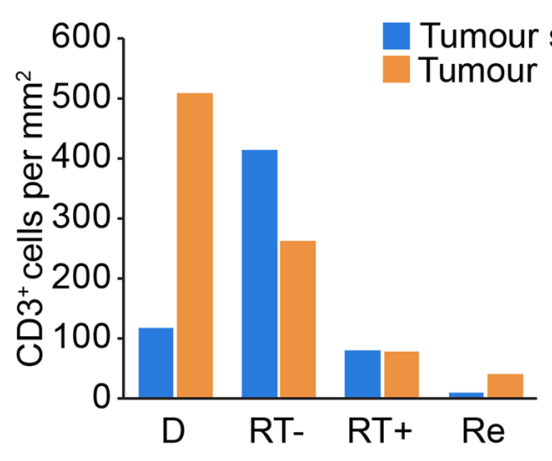

C

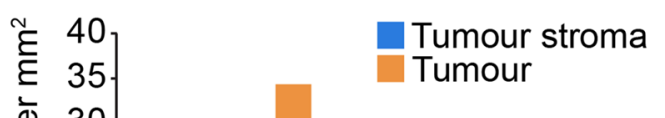

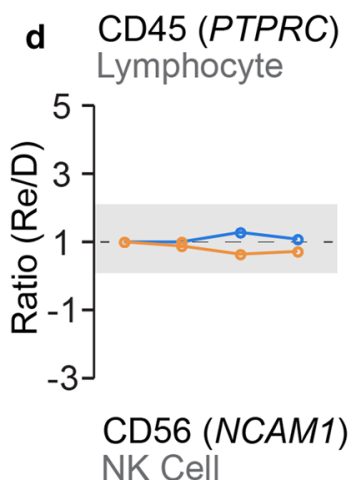

CD163

Macrophage

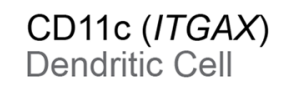

CD14
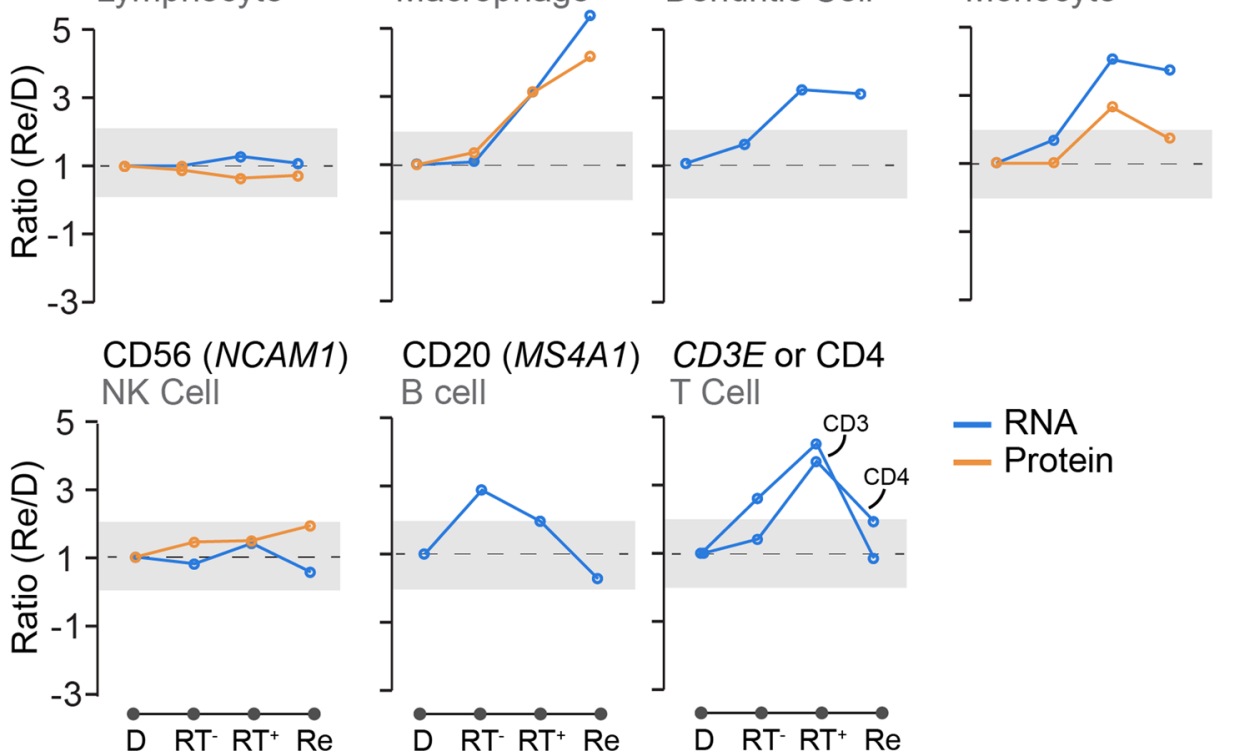

CD3E or CD4

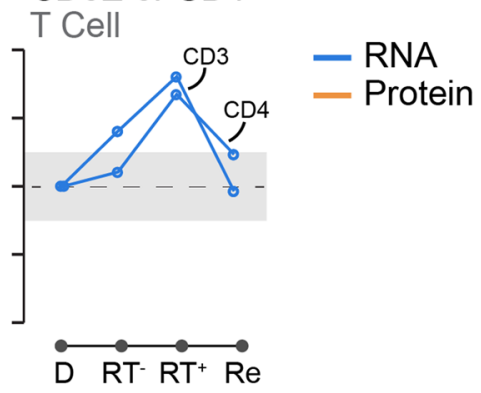

Fig. 4 Immuno-transcriptomic profiling reveals steady progression to immune-excluded phenotype. a Two-colour (pink and brown) DAB staining of the T cell marker CD3 (brown) and lymphocyte marker CD45 (pink) in four biopsies in tumour stroma and tumour zones. Scale bar indicates $100 \mu \mathrm{m}$. Quantification of b CD3+ and c CD45+ CD3- cell densities (per $\mathrm{mm}^{2}$ ) in each biopsy core tissue zone. Major treatments shown below. $\mathbf{d}$ Transcriptomic and proteomic analysis of immune cell marker expression changes over course of disease. Shaded areas indicates two-fold change threshold 
Table 1 Total surface area of tumor and tumor stroma zones in four patient samples

\begin{tabular}{llc}
\hline Sample & Tissue zone & $\begin{array}{l}\text { Surface } \\
\text { area } \\
\mathbf{( m m}^{\mathbf{2}} \mathbf{)}\end{array}$ \\
\hline Diagnostic & Tumor stroma & 1.4 \\
Pre-RT & Tumor & 4.78 \\
Post-RT & Tumor stroma & 2.25 \\
& Tumor & 8.63 \\
Recurrence & Tumor stroma & 5.11 \\
& Tumor & 9.73 \\
& Tumor stroma & 7.23 \\
\hline
\end{tabular}

\section{Common putative individual oncogenic drivers of NEPC are altered in recurrence}

Candidate analysis did not identify known drivers of lethal PC causing the treatment relapse in this case. As histopathological analysis strongly suggested NEPC biology, we directly tested if common NEPC biomarkers and drivers from the literature (Table 2) were dysregulated in the recurrence. This analysis revealed a strong signature of NEPC-upregulated transcripts (Fig. 5c) such as E2F1, AURKA, EZH2, TP53, ATR, PEG10, CRY1, MTOR. Common genomic losses in NEPC of RB1, PTEN, LTBP1, and $E R G$ were also reflected in corresponding RNA levels in the recurrent biopsy. These results strongly implicate known genomic and transcriptional drivers of NEPC in mediating the treatment-refractory disease course in this case.

\section{Recurrent disease shares known transcriptional characteristics of prototypical and atypical small cell prostate carcinoma, and NEPC}

We next performed ontology enrichment analysis using genesets that are over- or under-expressed in prototypical adenocarcinoma (AdPC), small cell prostatic carcinoma (SCPC), and neuroendocrine prostate cancer (NEPC) published by Tsai et al. [30]. For this purpose, we directly compared RNAseq data from the recurrence biopsy with the diagnostic specimen. This enabled a clear comparison of tumour profile without contamination from treatment variables. The results revealed a highly significant signature for both prototypical (Fig. 5d) and atypical SCPC (Fig. 5e) when compared to prototypical AdPC. In addition, contrast with another FFPE dataset compared SCPC with AdPC revealed strong signatures for SCPC as a whole (Fig. 5f). Finally, comparison with a curated genelist specific to NEPC (Meta-12 analysispresent in $>80 \%$ of NEPC) again strongly supported an NEPC transcriptional profile (Fig. 5g). Taken together these data confirm suspicion of NEPC biology. However, due to the known intratumoral heterogeneity within $\mathrm{NEPC}$, and the absence of appropriate comparative datasets, it is difficult to further resolve any of the specific NEPC subtypes any further.

\section{Aggressive recurrent disease is driven primarily by conventional molecular pathways}

Due to the particularly aggressive nature of the recurrent tumour, we sought to identify pathway over-activity (or inhibition) that was likely to be driving the disease. We therefore focused on comparing our data to canonical pathways using Gene Set Enrichment Analysis (GSEA). We interrogated both the more expansive Pathway Interaction Database (PID), and the highly curated MSigDB Hallmark (Hallmark) collections using both the transcriptional and proteomic datasets previously generated. Of particular interest were pathways regulated by known drivers (c.f. Fig. 5c), and those significant in both transcriptomic and proteomic analyses. It should be noted that the proteomic analysis (5,566 proteins) was restricted due to a lower number of identified molecules compared to transcriptomics (12,661 genes).

From the PID analysis, we confirmed that the AURORA Kinase A and B, MYC, ATR/ATM, RB1, E2F and p53 pathways were all upregulated at the transcriptional level (Fig. 6a). Amongst these, we also identified E2F and ATR upregulated at the proteomic level. Interestingly, we additionally identified the c-Myb transcriptional network upregulated at both the transcriptional and proteomic level. This proto-oncogene pathway has not yet been strongly linked with NEPC, but does have known hyperactivity in the castration-resistant PC adenocarcinoma

(See figure on next page.)

Fig. 5 Signature analysis uncovers aggressive SCPC and NEPC genetic profiles in recurrence. a Normalised expression heatmaps and hierarchical clustering of transcriptomic and proteomic changes in four biopsies. b Venn analysis of overlap in most increased (orange) and decreased (blue) molecules between transcriptomic and proteomic analysis. Common genes are listed below. $\mathbf{c}$ Normalised transcriptomic expression heatmap of commonly dysregulated genomic lesions in NEPC. (D-G) GSEA comparison transcriptomic changes in recurrence compared to diagnostic biopsy for published prostate cancer gene signatures of $\mathbf{d}$ prototypical SCPC, e atypical SCPC, $\mathbf{f}$ up- and down-regulated in SCPC versus AdPC and $\mathbf{g}$ up- and down-regulated in NEPC versus AdPC. SCPC: small cell prostate cancer, AdPC: prostate adenocarcinoma, NEPC: neuroendocrine prostate cancer, FDR: false discovery rate, NES: normalised enrichment score 
a Transcriptomics Proteomics

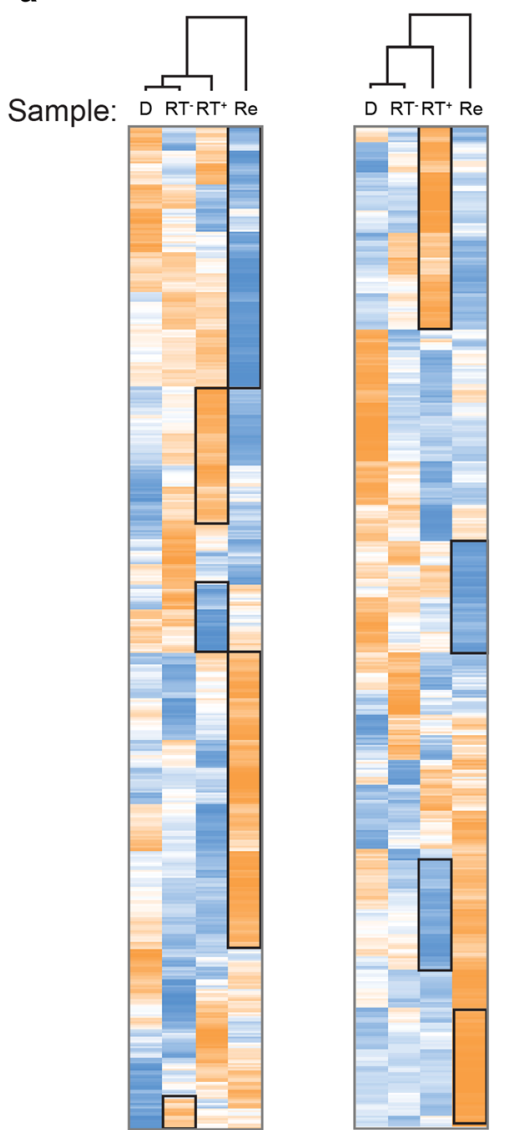

d Signature 1 - Prototypical SCPC

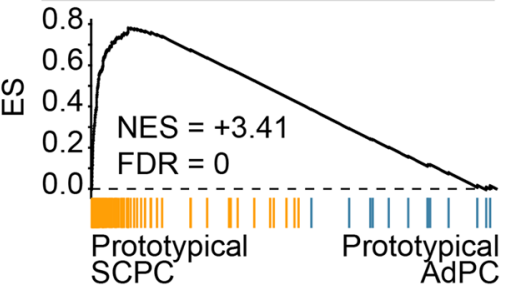

Upregulated

e Signature 2- Atypical SCPC

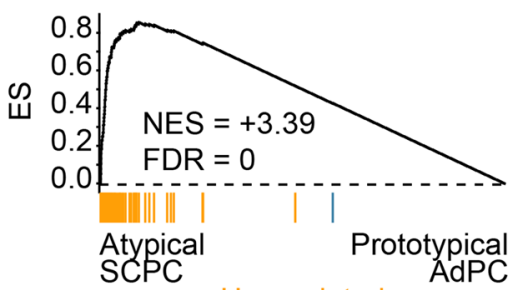

Upregulated
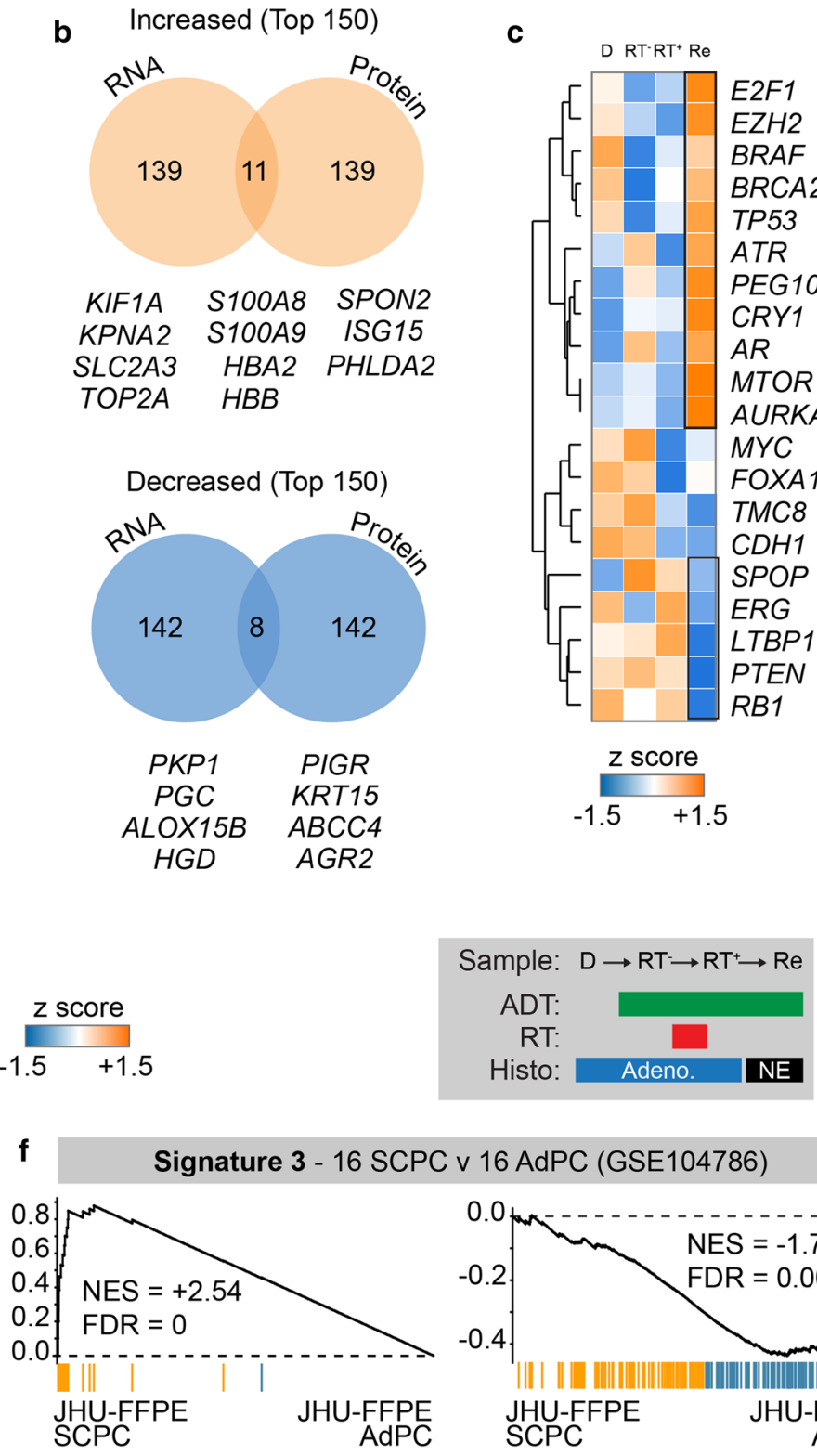

Upregulated

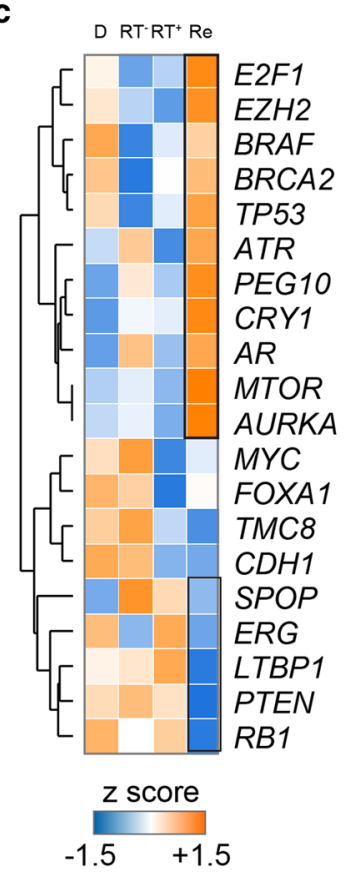

Histo: Adeno. NE

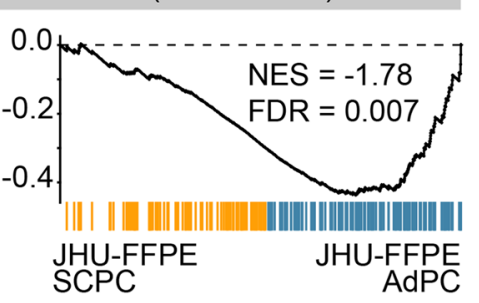

Downregulated

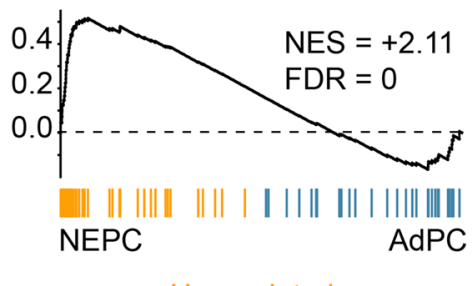

||| || ||||| || || | | | | || || | | | || || || || NEPC AdPC

\section{g Signature 4 - Meta-12 analysis (>80\% high grade NEPC)}

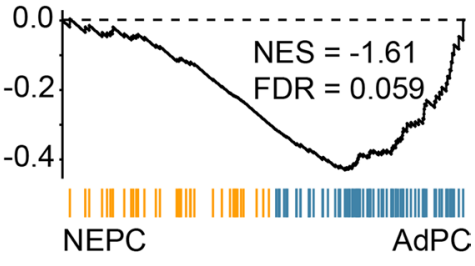


Table 2 Known genomic drivers of NEPC

\begin{tabular}{|c|c|c|}
\hline Gene & Product & References \\
\hline RB1 & RB Transcriptional Corepressor 1 & [19-21] \\
\hline PTEN & Phosphatase and tensin homolog & [20] \\
\hline CHD1 & Chromodomain Helicase DNA Binding Protein 1 & [19] \\
\hline TP53 & Tumor Protein P53 & {$[19,21]$} \\
\hline BRCA2 & BRCA2 DNA Repair Associated & [19] \\
\hline$A R$ & Androgen Receptor & {$[19,22]$} \\
\hline SPOP & Speckle Type BTB/POZ Protein & [19] \\
\hline TMC8 & Transmembrane Channel Like 8 & [19] \\
\hline FOXA1 & Forkhead Box A1 & [19] \\
\hline LTBP1 & Latent Transforming Growth Factor Beta Binding Protein 1 & [19] \\
\hline CRY1 & Cryptochrome Circadian Regulator 1 & [19] \\
\hline BRAF & B-Raf Proto-Oncogene, Serine/Threonine Kinase & [19] \\
\hline MYC & MYC Proto-Oncogene, BHLH Transcription Factor & {$[22,23]$} \\
\hline ERG & ETS Transcription Factor ERG & [24] \\
\hline AURKA & Aurora Kinase A & [22] \\
\hline E2F1 & E2F Transcription Factor 1 & [25] \\
\hline ATR & ATR Serine/Threonine Kinase & [26] \\
\hline MTOR & Mechanistic Target Of Rapamycin Kinase & [27] \\
\hline $\mathrm{EZH} 2$ & Enhancer Of Zeste 2 Polycomb Repressive Complex 2 Subunit & {$[22,28]$} \\
\hline PEG10 & Paternally Expressed 10 & [29] \\
\hline
\end{tabular}

cell C4-2 [31]. To provide additional evidence for the most important up- and down-regulated pathways, we performed a similar analysis using the Hallmark datasets. The results, shown in Fig. 6b, identified that E2F, MYC and p53 pathways, MTORC1, TNF-alpha, G2M checkpoint and complement pathways are robustly upregulated. Taken together, these two analyses suggested that Aurora kinase, E2F, MYC and p53 activity, are the putative drivers of the recurrent NEPC pathology.

\section{Whole genome sequencing reveals pervasive chromosomal alterations in recurrent tissue}

We identified a total of 18 single-copy copy number alterations (CNAs) in the recurrence which did not appear in any of the preceding samples (Fig. 7a). These changes included three whole chromosomal arm gains (4p, 5p and $8 q$ ) and five losses (4q, 8p, 13q, 17p and 18p). Three smaller amplifications and seven losses were observed elsewhere in the genome (Fig. 6; bottom panel). Due to the lower quality and quantity of FFPE material in preceding samples, the analysis could not automatically identify chromosomal changes in these samples. However, several of the recurrence sample CNAs could be observed in the diagnostic biopsy via processing (Fig. 7b). This included the $8 \mathrm{p}$ loss/8q gain, the $12 \mathrm{q} 14.2-24.32$ gain, and the 16q12.1-24.2 loss (marked in arrows). Interestingly, these were not as prominent in the
post-HDRBT biopsy-potentially due to the elimination of many of the tumours due to irradiation. Both $8 \mathrm{p}$ loss and $8 \mathrm{q}$ amplification are highly common genomic alterations in all stages of PC-with frequencies of 34\% and $\sim 29 \%$, respectively [32].

We also observed that several of the highly significant transcript and proteomic changes, as well as known NEPC markers, corresponded to these genomic changes in the recurrent tissue. For molecules already linked with NEPC, these included PEG10, RB1 and TP53. Whilst $R B 1$ exhibited the same directionality change as expected, the others were not as expected. For example, TP53 is located on the partially lost chromosomal arm 17p, yet exhibits transcriptional amplification. As previously mentioned, this chromosomal loss also appears to be an early event and visible in the diagnostic tissue. The discrepancy could be due to the subsequent mutation and stabilisation of TP53, which has been observed previously in small cell carcinomas of the prostate [33]. A similar observed was also made for PEG10, a molecule strongly associated with treatment-induced NEPC and metastasis [34] and also incidentally requires TP53 and $R B 1$ dysregulation [29]. As many of these putative NEPC markers are derived from genomics, rather than transcriptomics, it may be more informative to consider genomic CNAs than transcriptional change. 


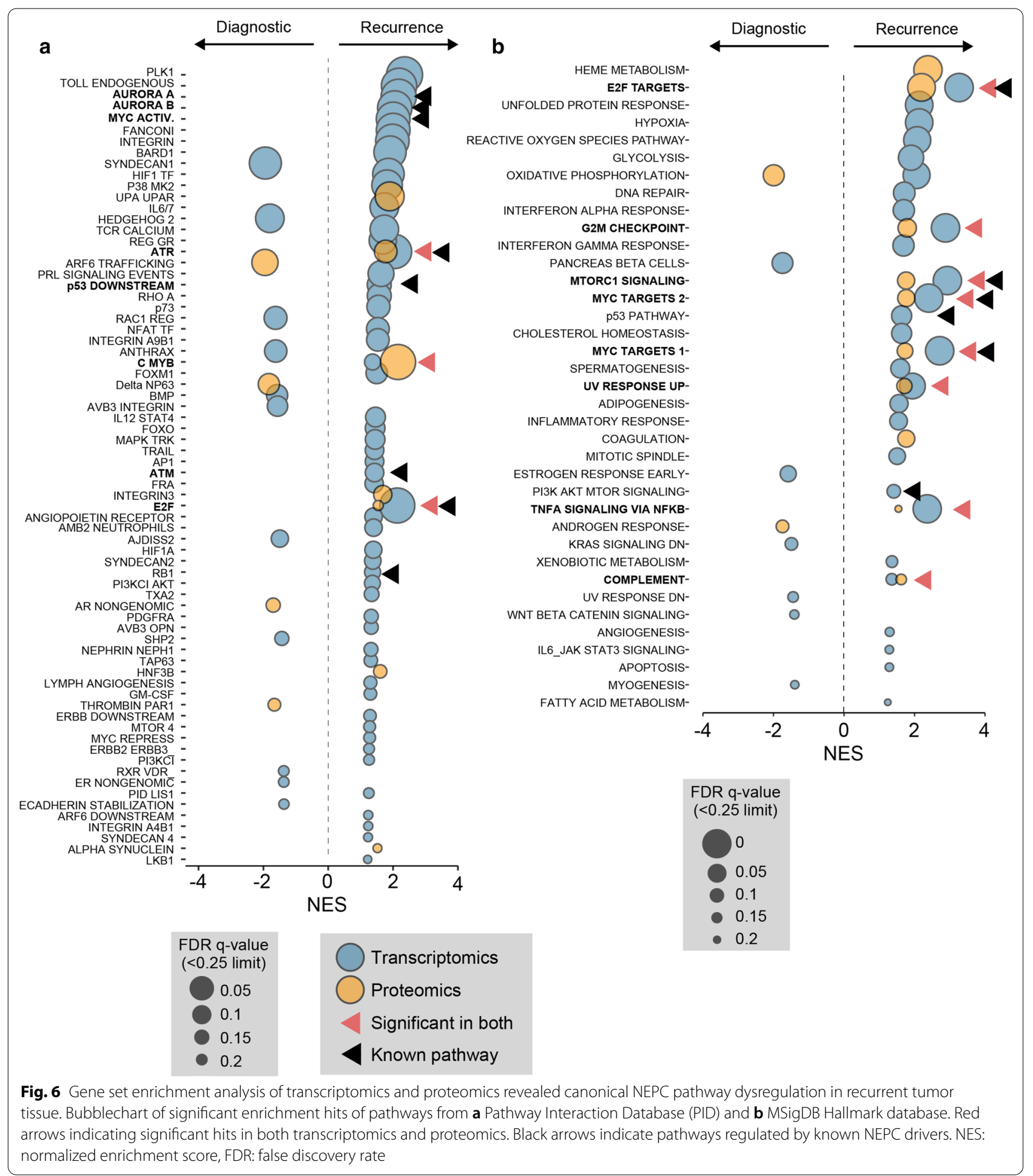

\section{Signatures of treatment delivery indicate a conventional response to radiation and ADT}

A conceivable source of treatment relapse is inefficacy of the two treatments used. Specifically, suboptimal treatment delivery or effect that leads to progression and the development of castrate resistance. To address this question, we used GSEA to compare the transcriptional and proteomic data at different treatment stages with 

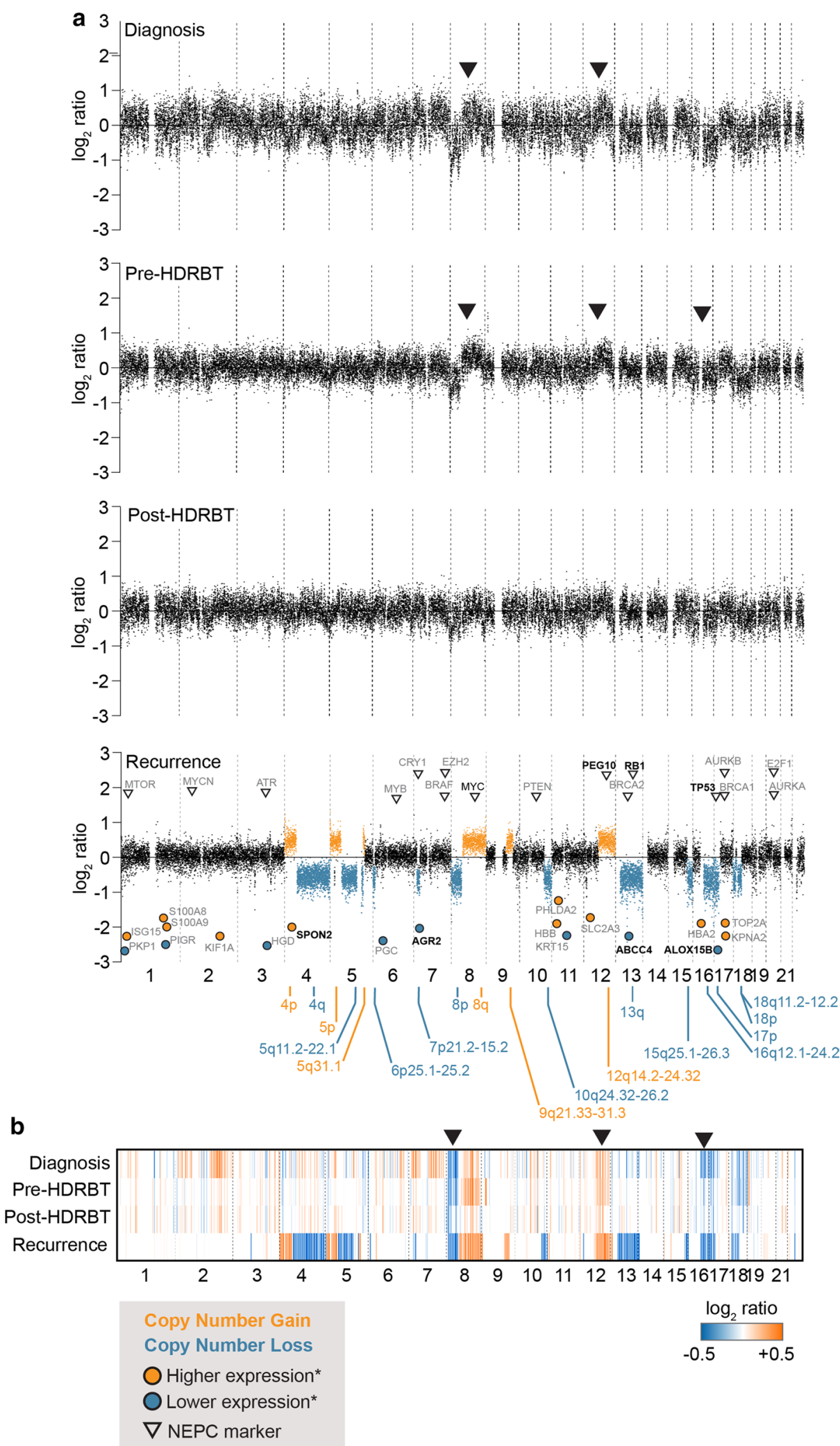

Fig. 7 Genomic copy number analysis (CNA) reveals both canonical and novel genomic alterations in emergent NEPC-like tumor. a Copy number scatterplot profile and $\mathbf{b}$ heatmap analysis of low coverage genomic sequencing and copy number analysis of four tissues in biopsy series. Recurrence panel overlayed with location of genes with expression changes from previous analyses, and known NEPC markers (black arrows) 
existing genesets associated with canonical biological responses to (i) ionizing radiation in $\mathrm{PC}$, and (ii) androgen deprivation. For radiation therapy, we drew upon an existing 300-gene (150 up and 150 down) signatures of both the transcriptional [4] (Fig. 8a) and the proteomic [3] radiation response in PC (Fig. 8b). In addition, we also compared with our pan-tissue dosimetric transcriptional signature and a corresponding non-dosimetric control signature (Fig. 8c) [35]. Taken together, the three analyses suggested that the case study exhibited a molecular response to therapeutic radiation in the prostate was consistent with an appropriate radiation treatment response.

The patient was also treated with androgen-deprivation in the form of goserelin prior to RT, and subsequent to radiation with additional bicalutamide, and then enzalutamide as CRPC emerged. To test if the initial ADT was effective, we contrasted the pre-RT sample (after 4 months of goserelin) with the baseline diagnostic biopsy (no ADT) at the transcriptional level using known androgen-regulated genes as a proxy for the absence of androgen signalling in the tissue-a consequence of androgen withdrawal. Androgen signalling was inferred using the Androgen Response Hallmark gene dataset and analysed using GSEA analysis (Fig. 8d; left panel). The results revealed the strong downregulation of the androgen pathway in the initial goserelin-treated sample-consistent with the use of goserelin and a biochemical PSA drop from 12.81 to $0.131 \mathrm{ng} / \mathrm{mL}$. This response was in stark contrast to that seen in the recurrence versus diagnostic analysis (Fig. 8d; right panel) where androgen signalling was no longer significantly altered-reinforcing the androgen-indifferent progression of the relapsed disease despite the administration of potent combination androgen suppression. Together, these results confirm that initial treatments impacted as expected at the molecular level, and do not suggest any atypical therapeutic responses were responsible for the development of aggressive disease.

\section{Discussion and conclusions}

In this study, we have presented an in-depth molecular case study of a fatal treatment-emergent NEPC-like prostate cancer. Here, initial diagnosis of T2c Gleason grade group 5 disease was subsequently treated with ADT and high-dose-rate brachytherapy/external beam RT. However, a poorly differentiated adenocarcinoma with NEPC differentiation became apparent following therapy and was resistant to increasingly aggressive ADT intervention (goserelin plus bicalutamide or enzalutamide) - subsequently progressing to death without evidence for metastasis via imaging. Our study has identified the cancer possessed some of the classical molecular, histological and genomic hallmarks of small cell PC and NEPC (e.g. Rb loss, AURKA gain etc.), however it did not exhibit many of the key clinical features such as low PSA rise and metastasis [36]. Conversely, we identified many novel molecules that are specifically expressed in the recurrent tissue which have strong links to more conventional PC pathologies and progression (e.g. $A B C C 4$ ). Many of these new molecules have limited known functional impact on $\mathrm{PC}$, and have instead been defined as novel prognostic or tumor markers. Otherwise, we have identified the c-Myb pathway as being of strong interest for this case of aggressive PC biology-with hyperactivation identified using pathway analysis. However, the literature describing the implication of this pathway in PC is limited to in vitro studies [31]. c-Myb-driven tumorigenesis has been linked with both pro-tumor and anti-oncogenic proliferation and differentiation functions, including in memory and effector $\mathrm{T}$ cell populations [37, 38]. Thus, c-Myb could be responsible for driving NEPClike recurrence pathologies through this mechanism, but is unlikely to dramatically affect the immune context given the lack of immune cells present. We identified aberrant expression of other novel molecules (e.g. TNF $\alpha$ pathway, ISG15 and PHLDA2) which are seemingly linked to NEPC development without any evidence for involvement in conventional prostate cancer. Further exploration of the c-Myb pathway and other unique molecules in NEPClike pathologies is warranted. We have also described the lack of an active immunological landscape within this this type of tumor, suggesting that it would not be amenable to any current form of immune-based therapy. Finally, we showed that the molecular response of the tumor to initial radiation- and androgen-based treatments was consistent with previously studied patients; advocating that treatment efficacy was not a factor in the emergence of aggressive disease.

The cataloguing of these molecular features in this interesting disease case will potentially provide valuable insight into other rare PC pathologies and improve treatment success. The distinctiveness of this case study warrants future work to determine if its biology is reflective of a broader group of patients with lethal locally-recurrent NEPC. 
Keam S.P. et al. IJROBP (2017)
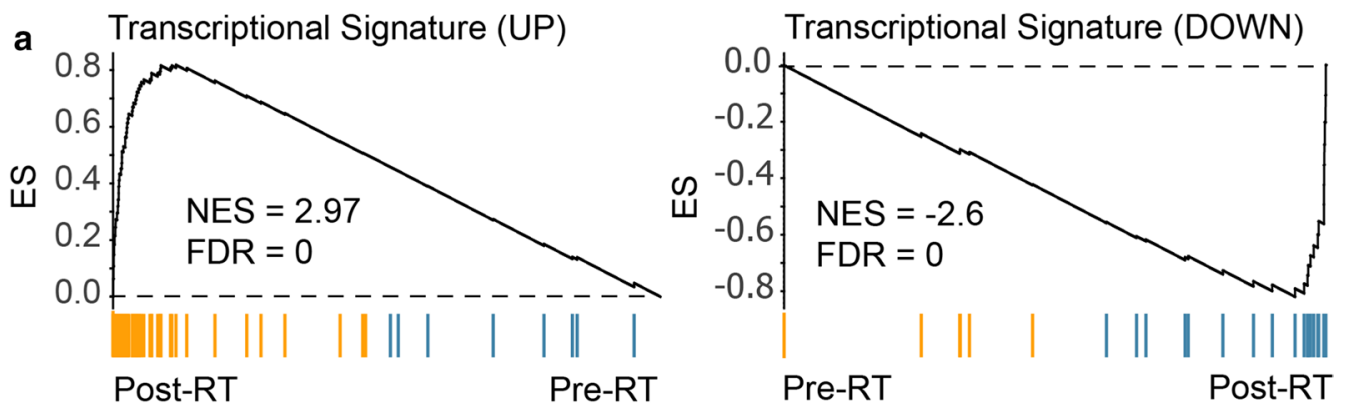

Keam S.P. et al. Prostate (2018)

b Proteomic Signature (UP)
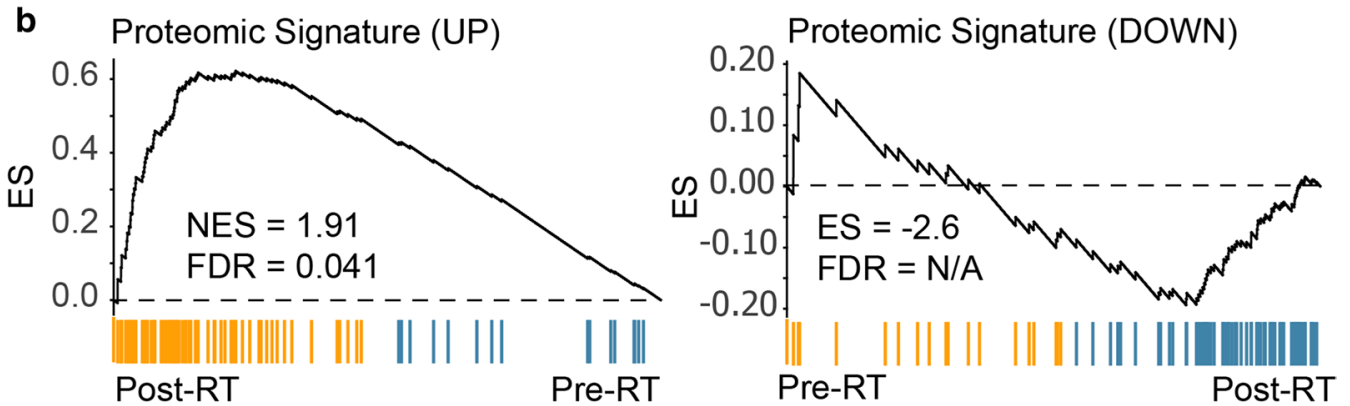

Keam S.P. et al. Rad. Env. Biophys. (2018)
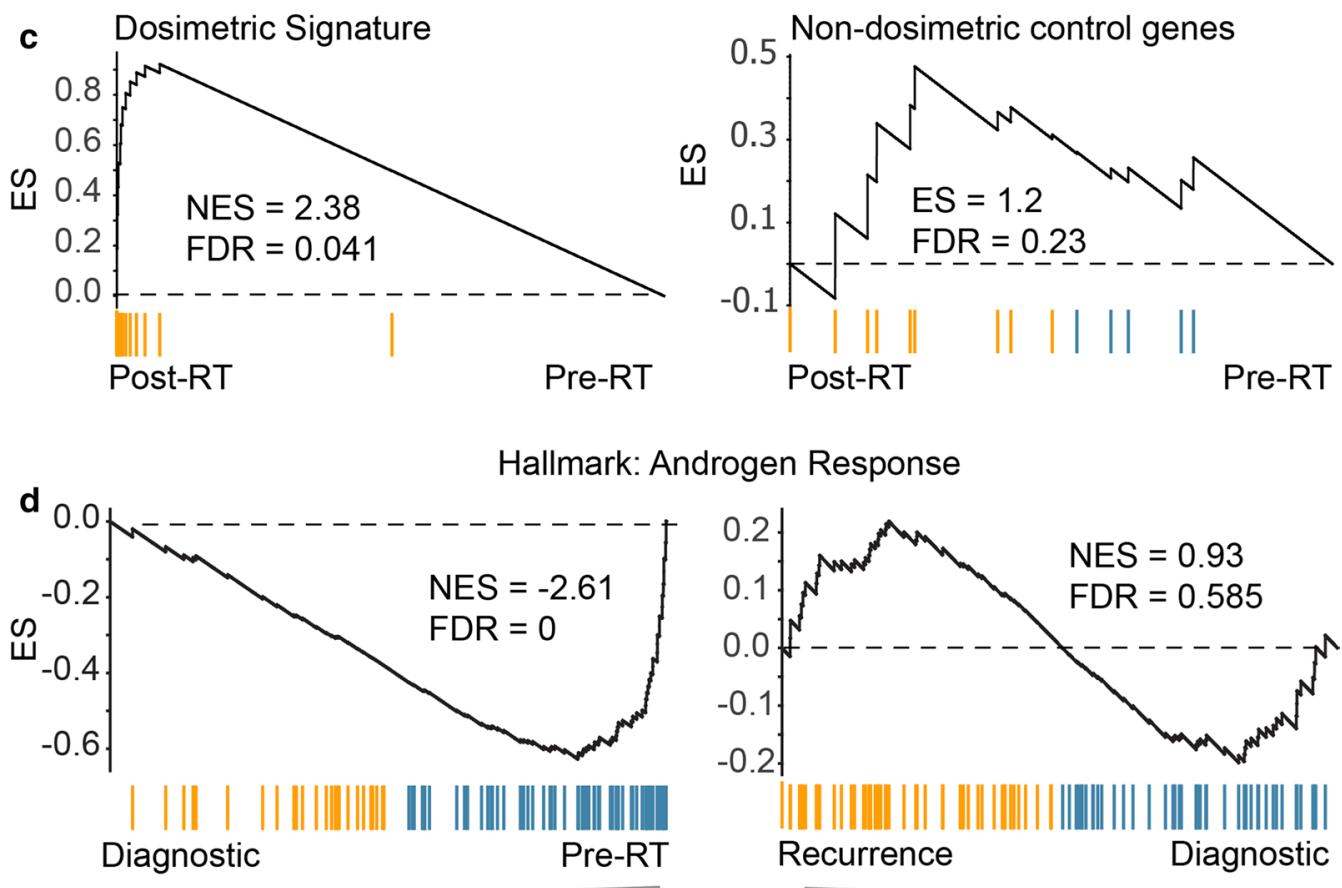

ADT

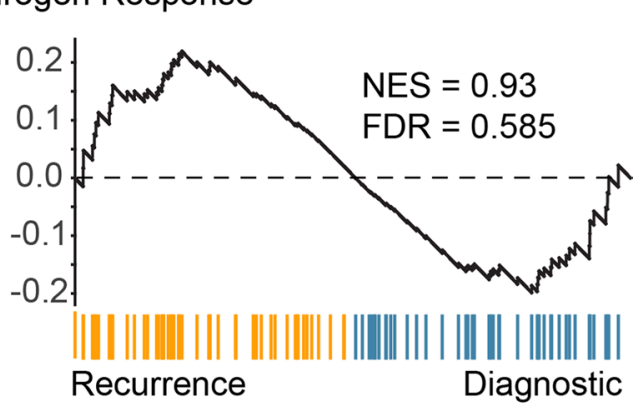

Fig. 8 GSEA analysis of biopsy pairs reveals conventional treatment responses. Gene set enrichment analysis was performed on biopsy pairs in order to identify the patterns associated with known radiation and androgen-associated gene and protein expression profiles. a Transcriptional and b proteomic response to HDRBT from Keam et al. 2017 and Keam et al. 2018a. c Dosimetric geneset response from Keam et al. 2018b. d MSigDb Hallmark Androgen Response signature. NES: normalised enrichment score. FDR: false discovery rate 


\section{Supplementary information}

Supplementary information accompanies this paper at https://doi. org/10.1186/s12894-020-00738-8.

Additional file 1. Supplementary methods for tissue collection, histology and staining, immune profiling, molecular characterization, genomic profiling, and bioinformatic approaches.

\section{Abbreviations}

NEPC: Neuroendocrine prostate cancer; PC: Prostate cancer; RT: Radiation therapy; AR: Androgen receptor; ADT: Androgen deprivation therapy; HDBRT: High dose-rate brachytherapy; PSA: Prostate specific antigen; FFPE: Formalinfixed paraffin-embedded; SYN: Synaptophysin; NSE: Neuron-specific enolase; CG: Chromogranin; IHC: Immunohistochemistry; DAB: 3,3'-Diaminobenzidine; CNA: Copy number alteration; CT: Computerized tomography; GSEA: Gene set enrichment analysis; PID: Protein interaction database; AdPC: Prostate adenocarcinoma; SCPC: Small cell prostatic carcinoma; TURP: Transurethral resection of prostate; PNI: Perineural invasion; FDR: False discovery rate; H\&E: Hematoxylin and eosin; NES: Normalized enrichment score.

\section{Acknowledgements}

We thank the Peter MacCallum Cancer Centre core facilities: Centre for Advanced Histology and Microscopy, Bioinformatics, and Molecular Genomics for their assistance with this study.

\section{Authors' contributions}

S.P.K., S.G.W. and Y.H. designed and conceived the study. S.P.K. performed experiments and analysed the results. R.B.S. designed, performed and analysed proteomics experiments. H.X.A.Y. perform immunohistological quantification experiments. F.C. assisted with bioinformatic analysis. C.M., D.B. and S.B.F. assisted with histological staining. S.P.K., S.G.W., S.H., P.J.N. and Y.H. drafted and critically revised the manuscript. All authors have read and approved the manuscript.

\section{Funding}

Tissue collection was partially funded by a Prostate Cancer Foundation USA (Creativity award) and a Victorian Cancer Agency award to S.G.W. Subsequent molecular analyses were supported by the PeterMac Foundation and an NHMRC Program Grant. Salary support was provided to S.P.K by a Cancer Council Victoria Grant-In-Aid. Core facilities were also supported by the Australian Cancer Research Foundation (ACRF).

\section{Availability of data and materials}

The datasets generated and/or analysed during the current study are available in the Mendeley repository with DOI identifier 10.17632/vmdrbgzdhw.1 at: https://data.mendeley.com/dtasets/vmdrbgzdhw/1. Raw data used for comparative analysis of SCPC and AdPC by Tsai et al., titled "Expression data from Neuroendocrine Prostate Cancer and Primary Small Cell Prostatic Carcinoma" can be obtained from the Gene Expression Omnibus accession GSE104786 and is directly available at: https://www.ncbi.nlm.nih.gov/geo/query/acc. cgi?acc $=$ GSE 104786

\section{Ethics approval and consent to participate}

The case study participant provided written consent covering tissue research as part of a prospective tissue collection study for prostate radiobiology research approved by the Human Research Ethics Committee at the Peter MacCallum Cancer Centre (PMCC; HREC approvals 10/68, 13/167, 19/32).

\section{Consent for publication}

The case study participant provided written consent for publication.

\section{Competing interests}

We have no conflicts of interest to disclose.

\section{Author details}

${ }^{1}$ Division of Radiation Oncology and Cancer Imaging, Peter MacCallum Cancer Centre, Melbourne, Australia. ${ }^{2}$ Tumor Suppression Laboratory, Peter MacCallum Cancer Centre, Melbourne, Australia. ${ }^{3}$ Cancer Immunology
Program, Peter MacCallum Cancer Centre, Melbourne, Australia. ${ }^{4}$ Pathology Department, Peter MacCallum Cancer Centre, Melbourne, Australia. ${ }^{5}$ Sir Peter MacCallum Department of Oncology, University of Melbourne, Melbourne, Australia. ${ }^{6}$ Monash Proteomics \& Metabolomics Facility, Monash University, Melbourne, Australia. ${ }^{7}$ Department of Biochemistry and Molecular Biology, Monash University, Melbourne, Australia.

Received: 20 August 2020 Accepted: 6 October 2020

Published online: 28 October 2020

\section{References}

1. Nakazawa M, Paller C, Kyprianou N. Mechanisms of therapeutic resistance in prostate cancer. Curr Oncol Rep. 2017;19(2):13.

2. Keam SP, et al. High dose-rate brachytherapy of localized prostate cancer converts tumors from cold to hot. J Immunother Cancer. 2020;8(1):e000792.

3. Keam SP, et al. Exploring the oncoproteomic response of human prostate cancer to therapeutic radiation using data-independent acquisition (DIA) mass spectrometry. Prostate. 2018a;78(8):563-75.

4. Keam SP, et al. The transcriptional landscape of radiation-treated human prostate cancer: analysis of a prospective tissue cohort. Int J Radiat Oncol Biol Phys. 2018b;100(1):188-98.

5. Labbe DP, et al. TOP2A and EZH2 provide early detection of an aggressive prostate cancer subgroup. Clin Cancer Res. 2017;23(22):7072-83.

6. Ren $\mathrm{L}$, et al. Copy number variation and high expression of DNA topoisomerase II alpha predict worse prognosis of cancer: a meta-analysis. J Cancer. 2018;9(12):2082-92.

7. Hille A, et al. Faecal calprotectin and lactoferrin values during irradiation of prostate cancer correlate with chronic radiation proctitis: results of a prospective study. Scand J Gastroenterol. 2009;44(8):939-46.

8. Jariwala $U$, et al. Identification of novel androgen receptor target genes in prostate cancer. Mol Cancer. 2007;6:39.

9. $\quad \operatorname{Vaz} C V$, et al. Androgens enhance the glycolytic metabolism and lactate export in prostate cancer cells by modulating the expression of GLUT1, GLUT3, PFK, LDH and MCT4 genes. J Cancer Res Clin Oncol. 2016;142(1):5-16.

10. Grupp K, et al. High nuclear karyopherin alpha 2 expression is a strong and independent predictor of biochemical recurrence in prostate cancer patients treated by radical prostatectomy. Mod Pathol. 2014:27(1):96-106

11. Qian X, et al. Spondin-2 (SPON2), a more prostate-cancer-specific diagnostic biomarker. PLoS ONE. 2012;7(5):e37225.

12. Lucarelli $G$, et al. Spondin-2, a secreted extracellular matrix protein, is a novel diagnostic biomarker for prostate cancer. J Urol. 2013;190(6):2271-7.

13. Maresh EL, et al. Differential expression of anterior gradient gene AGR2 in prostate cancer. BMC Cancer. 2010;10:680.

14. Montani M, et al. Multidrug resistance protein 4 (MRP4) expression in prostate cancer is associated with androgen signaling and decreases with tumor progression. Virchows Arch. 2013;462(4):437-43.

15. Feng $Y$, et al. Downregulation of 15-lipoxygenase 2 by glucocorticoid receptor in prostate cancer cells. Int J Oncol. 2010;36(6):1541-9.

16. Tang S, et al. Evidence that arachidonate 15-lipoxygenase 2 is a negative cell cycle regulator in normal prostate epithelial cells. J Biol Chem. 2002:277(18):16189-201.

17. Hassan Ml, Toor A, Ahmad F. Progastriscin: structure, function, and its role in tumor progression. J Mol Cell Biol. 2010;2(3):118-27.

18. Breuninger $S$, et al. Desmosomal plakophilins in the prostate and prostatic adenocarcinomas: implications for diagnosis and tumor progression. Am J Pathol. 2010;176(5):2509-19.

19. Beltran $\mathrm{H}$, et al. Divergent clonal evolution of castration-resistant neuroendocrine prostate cancer. Nat Med. 2016:22(3):298-305.

20. Tan HL, et al. Rb loss is characteristic of prostatic small cell neuroendocrine carcinoma. Clin Cancer Res. 2014;20(4):890-903.

21. Ku SY, et al. Rb1 and Trp53 cooperate to suppress prostate cancer lineage plasticity, metastasis, and antiandrogen resistance. Science. 2017;355(6320):78-83. 
22. Beltran $\mathrm{H}$, et al. Molecular characterization of neuroendocrine prostate cancer and identification of new drug targets. Cancer Discov. 2011;1(6):487-95

23. Thompson TC, Liu B, Li L. N-MYC regulation of DNA damage response in neuroendocrine prostate cancer: mechanistic insight and novel combination therapy approaches. Oncoscience. 2018;5(11-12):273-5.

24. Mounir Z, et al. TMPRSS2:ERG blocks neuroendocrine and luminal cell differentiation to maintain prostate cancer proliferation. Oncogene. 2015;34(29):3815-25.

25. Smith BA, et al. A basal stem cell signature identifies aggressive prostate cancer phenotypes. Proc Natl Acad Sci U S A. 2015;112(47):E6544-52

26. Yin $\mathrm{Y}$, et al. N-Myc promotes therapeutic resistance development of neuroendocrine prostate cancer by differentially regulating miR-421/ATM pathway. Mol Cancer. 2019;18(1):11.

27. Kanayama $\mathrm{M}$, et al. Hyperactive $\mathrm{mTOR}$ induces neuroendocrine differentiation in prostate cancer cell with concurrent up-regulation of IRF1. Prostate. 2017;77(15):1489-98

28. Shan J, et al. Targeting Wnt/EZH2/microRNA-708 signaling pathway inhibits neuroendocrine differentiation in prostate cancer. Cell Death Discov. 2019;5:139.

29. Akamatsu S, et al. The placental gene PEG10 promotes progression of neuroendocrine prostate Cancer. Cell Rep. 2015;12(6):922-36.

30. Tsai HK, et al. Gene expression signatures of neuroendocrine prostate cancer and primary small cell prostatic carcinoma. BMC Cancer 2017;17(1):759.

31. Srivastava SK, et al. Myb overexpression overrides androgen depletioninduced cell cycle arrest and apoptosis in prostate cancer cells, and confers aggressive malignant traits: potential role in castration resistance. Carcinogenesis. 2012;33(6):1149-57.
32. El Gammal AT, et al. Chromosome $8 p$ deletions and $8 q$ gains are associated with tumor progression and poor prognosis in prostate cancer. Clin Cancer Res. 2010;16(1):56-64.

33. Kadakia KC, et al. Comprehensive serial molecular profiling of an " $\mathrm{N}$ of 1 " exceptional non-responder with metastatic prostate cancer progressing to small cell carcinoma on treatment. J Hematol Oncol. 2015;8:109.

34. Kim S, et al. PEG10 is associated with treatment-induced neuroendocrine prostate cancer. J Mol Endocrinol. 2019;63(1):39-49.

35. Keam SP, et al. Biodosimetric transcriptional and proteomic changes are conserved in irradiated human tissue. Radiat Environ Biophys. 2018c;57(3):241-9.

36. Conteduca $V$, et al. Clinical features of neuroendocrine prostate cancer. Eur J Cancer. 2019;121:7-18.

37. George OL, Ness SA. Situational awareness: regulation of the myb transcription factor in differentiation, the cell cycle and oncogenesis. Cancers (Basel). 2014;6(4):2049-71.

38. Gautam S, et al. The transcription factor c-Myb regulates CD8(+) T cell stemness and antitumor immunity. Nat Immunol. 2019;20(3):337-49.

\section{Publisher's Note}

Springer Nature remains neutral with regard to jurisdictional claims in published maps and institutional affiliations.
Ready to submit your research? Choose BMC and benefit from:

- fast, convenient online submission

- thorough peer review by experienced researchers in your field

- rapid publication on acceptance

- support for research data, including large and complex data types

- gold Open Access which fosters wider collaboration and increased citations

- maximum visibility for your research: over $100 \mathrm{M}$ website views per year

At BMC, research is always in progress.

Learn more biomedcentral.com/submissions 